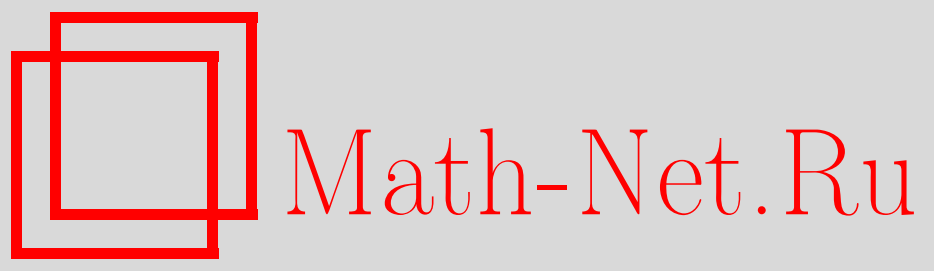

А. А. Славнов, Квантование неабелевых калибровочных полей вне рамок теории возмущений, ТМФ, 2014, том 181, номер 1, 200-205

DOI: https://doi.org/10.4213/tmf8698

Использование Общероссийского математического портала Math-Net.Ru подразумевает, что вы прочитали и согласны с пользовательским соглашением http://www . mathnet.ru/rus/agreement

Параметры загрузки:

IP : 54.224 .60 .19

26 апреля 2023 г., $17: 25: 41$

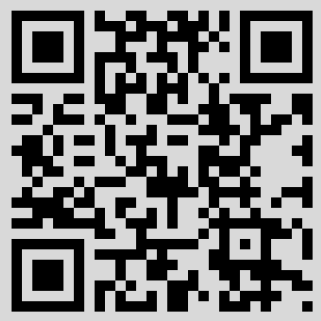




\title{
КВАНТОВАНИЕ НЕАБЕЛЕВЫХ КАЛИБРОВОЧНЫХ ПОЛЕЙ ВНЕ РАМОК ТЕОРИИ ВОЗМУЩЕНИЙ
}

\begin{abstract}
Предложена новая процедура квантования калибровочных полей, позволяющая избежать неоднозначности Грибова и, следовательно, применимая вне рамок теории возмущений. Эта процедура применяется для построения калибровочно-инвариантной инфракрасной регуляризации теории Янга-Миллса.
\end{abstract}

Ключевые слова: калибровочные поля, теория возмущений, инфракрасные расходимости.

DOI: $10.4213 / \operatorname{tmf} 8698$

\section{1. ВВЕДЕНИЕ}

Решающий шаг в построении процедуры квантования неабелевых калибровочных полей был сделан Фаддеевым и Поповым [1] и независимо в работе [2]. Однако, строго говоря, процедура квантования, построенная в этих работах, применима лишь в рамках теории возмущений.

Грибов заметил [3], что кулоновская калибровка $\partial_{i} A_{i}=0$ не позволяет однозначно выбрать представителя в классе калибровочно-эквивалентных конфигураций, поскольку условие

$$
\partial_{i} A_{i}^{\Omega}=0
$$

рассматриваемое как уравнение на элементы калибровочной группы $\Omega$ на поверхности $\partial_{i} A^{i}=0$, для достаточно больших $A$ имеет нетривиальные решения, быстро убывающие на пространственной бесконечности. Этот результат был обобщен Зингером [4] на произвольные калибровочные условия.

В рамках теории возмущений, т. е. для достаточно малых $A$, уравнение (1) имеет только тривиальное решение. Следовательно, в этом случае неоднозначность Грибова отсутствует. Однако вне теории возмущений эта неоднозначность существует, что ставит под сомнение законность стандартной процедуры канонического квантования неабелевых калибровочных теорий. Эта проблема исследовалась многими авторами (см., например, работу [5]), но, по моему мнению, вопрос остается нерешенным.

* Математический институт им. В. А. Стеклова РАН, Москва, Россия. E-mail: slavnov@mi.ras.ru 
Инфракрасные свойства теории Янга-Миллса также неясны. Добавление массы к лагранжиану поля Янга-Миллса нарушает калибровочную инвариантность теории. Можно попытаться ввести массу калибровочно-инвариантным образом, пользуясь формализмом Штюкельберга. При этом, однако, теряется перенормируемость, и в пределе, когда масса стремится к нулю, мы не получаем безмассовую теорию Янга-Миллса [6]. Если начинать с модели Хиггса, то в пределе нулевой массы мы получим теорию поля Янга-Миллса, взаимодействующего с безмассовым скаляром. Калибровочно инвариантная инфракрасная регуляризация поля Янга-Миллса до сих пор была неизвестна.

\section{2. ОПИСАНИЕ МОДЕЛИ}

Мы рассматриваем модель, которая описывается классическим лагранжианом [7]-[9]

$$
L=-\frac{1}{4} F_{\mu \nu}^{a} F_{\mu \nu}^{a}+\left(D_{\mu} \varphi\right)^{*}\left(D_{\mu} \varphi\right)-\alpha^{2}\left(D_{\mu} \chi\right)^{*}\left(D_{\mu} \chi\right)+i\left[\left(D_{\mu} b\right)^{*}\left(D_{\mu} e\right)-\left(D_{\mu} e\right)^{*}\left(D_{\mu} b\right)\right] .
$$

В целях экономии места мы будем работать с моделью, инвариантной относительно калибровочной группы $S U(2)$. Здесь $F_{\mu \nu}^{a}$ - стандартный тензор кривизны для поля Янга-Миллса. Скалярные поля $\varphi, \chi, b, e$ образуют комплексные $S U(2)$-дублеты, которые в терминах эрмитовых компонент имеют вид

$$
c=\left(\frac{i c_{1}+c_{2}}{\sqrt{2}}, \frac{c_{0}-i c_{3}}{\sqrt{2}}\right),
$$

где $c$ обозначает любой из дублетов. Считается, что поля $\varphi$ и $\chi$ коммутируют, а поля $e$ и $b$ антикоммутируют. В формуле (2) $D_{\mu}$ обозначает обычную ковариантную производную, поэтому лагранжиан (2) калибровочно-инвариантен. Заметим, что благодаря знаку минус перед лагранжианом полей $\chi$ эти поля обладают отрицательной энергией.

Сделаем в лагранжиане (2) следующие сдвиги полей:

$$
\varphi \rightarrow \varphi+g^{-1} \widehat{m}, \quad \chi \rightarrow \chi-\alpha g^{-1} \widehat{m}, \quad \widehat{m}=(0, m),
$$

где $m$-постоянный параметр. Возникающая при этом теория, очевидно, калибровочно-инвариантна при любом $\alpha$, однако вид калибровочных преобразований и лагранжиана, разумеется, зависит от выбора $\alpha$. При $\alpha=1$ члены, квадратичные по $m$, которые возникают благодаря сдвигам полей $\varphi$ и $\chi$, взаимно компенсируются, и лагранжиан принимает вид

$$
\begin{aligned}
L= & -\frac{1}{4} F_{\mu \nu}^{a} F_{\mu \nu}^{a}+\left(D_{\mu} \varphi\right)^{*}\left(D_{\mu} \varphi\right)-\left(D_{\mu} \chi\right)^{*}\left(D_{\mu} \chi\right)+ \\
& +g^{-1}\left[\left(D_{\mu} \varphi\right)^{*}+\left(D_{\mu} \chi\right)^{*}\right]\left(D_{\mu} \widehat{m}\right)+g^{-1}\left(D_{\mu} \widehat{m}\right)^{*}\left[D_{\mu} \varphi+D_{\mu} \chi\right]+ \\
& +i\left[\left(D_{\mu} b\right)^{*}\left(D_{\mu} e\right)-\left(D_{\mu} e\right)^{*}\left(D_{\mu} b\right)\right]
\end{aligned}
$$

Как и раньше, этот лагранжиан описывает при $\alpha=1$ безмассовые векторные частицы. Важно отметить, что лагранжиан (3) калибровочно-инвариантен при любом $\alpha$. Ниже будет показано, что при $\alpha=1$ матрица рассеяния, описываемая лагранжианом (3), совпадает с матрицей рассеяния обычной теории Янга-Миллса. Конечно, 
это совпадение формальное, так как при $\alpha=1$ обе матрицы не существуют из-за инфракрасных расходимостей, но имеет смысл говорить об обращении в ноль амплитуд переходов из физических состояний, отвечающих трехмерно поперечным компонентам поля Янга-Миллса, в нефизические. Таким образом, можно сказать, что предел $\alpha \rightarrow 1$ отвечает снятию инфракрасной регуляризации и возвращению к обычной теории Янга-Миллса. При $\alpha \neq 1$ векторное поле массивно, и инфракрасные расходимости отсутствуют. При этом теория на всех этапах калибровочноинвариантна.

Лагранжиан (3), очевидно, инвариантен относительно “сдвинутых" калибровочных преобразований, которые в терминах эрмитовых компонент имеют вид

$$
\begin{aligned}
\delta A_{\mu}^{a} & =\partial_{\mu} \eta^{a}-g \epsilon^{a b c} A_{\mu}^{b} \eta^{c} \\
\delta \varphi_{a}^{+} & =-m \eta^{a} \frac{1-\alpha}{2}-\frac{g}{2} \epsilon^{a b c} \varphi_{b}^{+} \eta^{c}-\frac{g}{2} \varphi_{0}^{+} \eta^{a} \\
\delta \varphi_{a}^{-} & =-m \eta^{a} \frac{1+\alpha}{2}-\frac{g}{2} \epsilon^{a b c} \varphi_{b}^{-} \eta^{c}-\frac{g}{2} \varphi_{0}^{-} \eta^{a} \\
\delta \varphi_{0}^{+} & =\frac{g}{2} \varphi_{a}^{+} \eta^{a} \\
\delta \varphi_{0}^{-} & =\frac{g}{2} \varphi_{a}^{-} \eta^{a} \\
\delta b^{a} & =-\frac{g}{2} \epsilon^{a d c} b^{d} \eta^{c}-\frac{g}{2} b^{0} \eta^{a}, \\
\delta e^{a} & =-\frac{g}{2} \epsilon^{a d c} e^{d} \eta^{c}-\frac{g}{2} e^{0} \eta^{a}, \\
\delta b^{0} & =\frac{g}{2} b^{a} \eta^{a} \\
\delta e^{0} & =\frac{g}{2} e^{a} \eta^{a} .
\end{aligned}
$$

Здесь использованы очевидные обозначения

$$
\varphi_{\beta}^{ \pm}=\frac{\varphi_{\beta} \pm \chi_{\beta}}{\sqrt{2}} .
$$

Помимо калибровочной инвариантности, лагранжиан (3) при $\alpha=1$ обладает также инвариантностью относительно преобразований суперсимметрии

$$
\begin{aligned}
\delta \varphi(x) & =i \epsilon b(x), & \delta \chi(x) & =-i \epsilon b(x), \\
\delta e(x) & =\epsilon[\varphi(x)+\chi(x)], & \delta b(x) & =0,
\end{aligned}
$$

где $\epsilon$ - постоянный антикоммутирующий параметр. Ниже мы увидим, что инвариантность относительно этих преобразований обеспечивает унитарность теории в пространстве, которое включает только физические возбуждения полей Янга-Миллса. При этом существенную роль играет не явный вид взаимодействия, а его свойства симметрии. В принципе допустимы любые контрчлены, сохраняющие калибровочную инвариантность и суперсимметрию теории. При $\alpha \neq 1$ инвариантность относительно преобразований (5) нарушена, что приводит к неунитарности теории.

Как видно из закона преобразований (4), поля $\varphi_{-}^{a}, \varphi_{+}^{a}$ сдвигаются при этих преобразованиях на произвольную функцию, что позволяет выбрать калибровку $\varphi_{-}^{a}=0$. 
Эта калибровка является явно лоренц-инвариантной и в то же время алгебраической, и это дает возможность использовать ее как при вычислениях по теории возмущений, так и вне нее.

Как уже отмечалось, при $\alpha \neq 1$ матрица рассеяния неунитарна. Это может показаться странным, так как обычно калибровочная инвариантность является достаточным условием перенормируемости и позволяет переходить от унитарной калибровки к перенормируемой. В нашем случае унитарная калибровка отсутствует. Даже в калибровке $\varphi_{-}^{a}=0$ присутствуют духовые нефизические состояния.

Каноническое квантование в калибровке $\varphi_{-}^{a}=0$ требует введения локальных духовых полей. Фиксирующий калибровку член имеет вид

$$
s \int d^{4} x\left(\bar{c}^{a} \varphi_{a}^{-}\right)=\int d^{4} x\left(\lambda^{a} \varphi_{-}^{a}+\bar{c}^{a} M_{a b} c^{b}\right) .
$$

Будучи локальными, духовые поля не влияют на унитарность матрицы рассеяния, поэтому в дальнейшем мы будем их игнорировать.

Налагая условие $\varphi_{-}^{a}=0$, мы нарушаем инвариантность относительно преобразований (5). При этом, поскольку переход от одной калибровки к другой может быть осуществлен с помощью калибровочного преобразования, а в кулоновской калибровке инвариантность относительно преобразований (5) при $\alpha=1$ имеет место, можно найти функцию, осуществляющую переход от одной калибровки к другой, решая уравнение

$$
\int d^{4} x\left(\lambda^{a} \partial_{i} A_{i}\right)=\int d^{4} x\left(\lambda^{a}\left(\varphi_{-}^{a}\right)^{\Omega}\right) .
$$

Так как мы рассматриваем рассеяние асимптотических состояний, достаточно решить это уравнение в нулевом порядке по константе связи. Отсюда находим

$$
\eta^{a}=\frac{\partial_{i} A_{i}^{a}-\varphi_{-}^{a}}{m} .
$$

Следовательно, $\eta^{a}$ преобразуется при преобразованиях суперсимметрии следующим образом:

$$
\eta^{a} \rightarrow \eta^{a}-i \frac{\sqrt{2} \epsilon b^{a}}{m} .
$$

Преобразование поля $A_{\mu}^{a}$ имеет вид

$$
A_{\mu}^{a} \rightarrow A_{\mu}^{a}+i \frac{\sqrt{2} \epsilon b^{a}}{m} .
$$

Асимптотические поля преобразуются как

$$
\begin{array}{cll}
\delta A_{\mu}^{a}=i \sqrt{2} \partial_{\mu} b^{a} m^{-1} \epsilon, \\
\delta \phi_{-}^{a}=0, \quad \delta \phi_{-}^{0}=i b^{0} \epsilon, & \delta e^{a}=\varphi_{+}^{a} \epsilon, & \delta e^{0}=\varphi_{+}^{0} \epsilon, \\
\delta \phi_{+}^{a}=0, & \delta \phi_{+}^{0}=0, \quad \delta b^{a}=0, & \delta b^{0}=0 .
\end{array}
$$

Инвариантность относительно этих преобразований по теореме Нётер приводит к существованию асимптотического сохраняющегося заряда, который можно представить в виде суммы $\widehat{Q}^{0}=\widehat{Q}_{\mathrm{B}}^{0}+\widehat{Q}_{\mathrm{S}}^{0}$, где

$$
\widehat{Q}_{\mathrm{S}}^{0}|\psi\rangle_{\text {as }}=0, \quad \widehat{Q}_{\mathrm{B}}^{0}|\psi\rangle_{\text {as }}=0 .
$$


Операторы $\widehat{Q}_{\mathrm{B}}^{0}, \widehat{Q}_{\mathrm{S}}^{0}$ имеют вид

$$
\begin{aligned}
& \widehat{Q}_{\mathrm{B}}^{0}=\int d^{3} x\left[m^{-1}\left(\partial_{i} A_{0}-\partial_{0} A_{i}\right) \partial_{i} b^{a}-\varphi_{a}^{+} \partial_{0} b^{a}\right], \\
& \widehat{Q}_{\mathrm{S}}^{0}=\int d^{3} x\left(\partial_{0} b \varphi_{+}^{0}+b_{0} \partial_{0} \varphi_{0}^{+}\right) .
\end{aligned}
$$

Оператор $\widehat{Q}_{\mathrm{B}}^{0}$ совпадает с БРСТ-зарядом в теории Янга-Миллса в поперечной калибровке, если отождествить поля $b^{a}, e^{a}$ с духами Фаддеева-Попова, а поле $\varphi_{+}^{a}-$ с соответствующим множителем Лагранжа. Оператор $\widehat{Q}_{\mathrm{S}}^{0}$ обеспечивает отсутствие в асимптотических состояниях частиц, отвечающих полям $b_{0}, \varphi_{0}^{ \pm}, b_{0}, e_{0}$. Это можно доказать обычным образом, вводя оператор числа нефизических частиц, отвечающих полям $b_{0}, \varphi_{0}^{ \pm}, b_{0}, e_{0}$. Его можно представить в виде антикоммутатора оператора $\widehat{Q}_{\mathrm{S}}^{0}$ с оператором $\widehat{K}$,

$$
\widehat{K}=\int d^{3} k\left[\left(e_{0}(k)\right)^{*} \varphi_{0}^{-}(k)+\left(\varphi_{0}^{-}\right)^{*}(k) e_{0}(k)\right] .
$$

Любой вектор, содержащий по крайней мере одну из вышеуказанных нефизических частиц, можно представить в виде

$$
|\psi\rangle=\frac{1}{N}\left(\widehat{Q}^{0} \widehat{K}+\widehat{K} \widehat{Q}^{0}\right)|\psi\rangle
$$

где $N$ - число нефизических частиц. Следовательно любой вектор, аннигилируемый операторами $\widehat{Q}_{\mathrm{B}}^{0}, \widehat{Q}_{s}^{0}$, имеет вид $|\psi\rangle_{\text {as }}=|\psi\rangle_{\mathrm{tr}}+|N\rangle$, где вектор $|\psi\rangle_{\text {tr }}$ содержит только поперечные возбуждения, а вектор $|N\rangle$ имеет нулевую норму.

\section{3. ОБСУЖДЕНИЕ РЕЗУЛЬТАТОВ}

Как видно, даже при $\alpha=1$ спектр модифицированной теории содержит дополнительные по сравнению со стандартной формулировкой духовые состояния. Духовые возбуждения суть поля $\varphi_{ \pm}, b, e$, продольные и временны́е компоненты поля Янга-Миллса. Физические состояния включают трехмерно поперечные компоненты поля Янга-Миллса.

Рассмотренной выше симметрии достаточно, чтобы доказать перенормируемость теории и унитарность матрицы рассеяния в подпространстве, содержащем только физические (трехмерно поперечные) поляризации поля Янга-Миллса. Матрица рассеяния при $\alpha \neq 1$ неунитарна в физическом подпространстве. Это означает, что введение отличного от единицы параметра $\alpha$ осуществляет калибровочно-инвариантную инфракрасную регуляризацию.

Предлагаемая формулировка позволяет по-новому взглянуть на проблему существования нетривиальных солитонных решений типа магнитного монополя в чистой теории Янга-Миллса. Обычные аргументы, исключающие существование солитонных решений в теории Янга-Миллса, неприменимы к данной формулировке. Разумеется, надо доказать существование таких решений. Мы предполагаем вернуться к обсуждению этой проблемы в одной из следующих публикаций.

Благодарности. Настоящая работа частично финансово поддержана РФФИ (грант № 13-01-12405-офи_м) и программой РАН "Нелинейная динамика". 


\section{Список литературы}

[1] L. D. Faddeev, V. N. Popov, Phys. Lett. B, 25:1 (1967), 29-30.

[2] B. S. DeWitt, Phys. Rev., 160:5 (1967), 1113-1148; 162:5 (1962), 1195-1239.

[3] V.N. Gribov, Nucl. Phys. B, 139:1-2 (1978), 1-19.

[4] I. Singer, Commun. Math. Phys., 60:1 (1978), 7-12.

[5] D. Zwanziger, Nucl. Phys. B, 321:3 (1989), 591-604; 323:3 (1989), 513-544.

[6] А. А. Славнов, Л. Д. Фаддеев, ТМФ, 3:1 (1970), 18-23.

[7] A. A. Slavnov, JHEP, 08 (2008), 047, 11 pp.

[8] А. А. Славнов, ТМФ, 161:2 (2009), 204-211.

[9] A. Quadri, A. A. Slavnov, JHEP, 07 (2010), 087, 22 pp.

Поступила в редакцию 10.04.2014 